\title{
Ensaios toxicológicos clínicos da casca do maracujá-amarelo (Passiflora edulis, f. flavicarpa), como alimento com propriedade de saúde
}

\author{
Josimar dos Santos Medeiros, ${ }^{*}, 1,2$ Margareth de Fátima F. Melo Diniz, ${ }^{1}$ Armando Ubirajara \\ Oliveira Sabaa Srur, ${ }^{3}$ Marcelo Barbosa Pessoa, ${ }^{2}$ Maria Aparecida Alves Cardoso, ${ }^{4}$ \\ Danielle Franklin de Carvalho ${ }^{4}$
}

\author{
${ }^{1}$ Laboratório de Tecnologia Farmacêutica, Universidade Federal da Paraíba, Caixa Postal 5009, 58051-900 \\ João Pessoa-PB, Brasil, \\ ${ }^{2}$ Departamento de Farmácia, Universidade Estadual da Paraíba, Rua Edson do Ó 86, Centenário, 58107-733 \\ Campina Grande-PB, Brasil, \\ ${ }^{3}$ Departamento de Nutrição Básica Experimental, Instituto de Nutrição, Universidade Federal do Rio de Janeiro, \\ Cidade Universitária, 21949-9000 Rio de Janeiro-RJ, Brasil, \\ ${ }^{4}$ Núcleo de Estudos e Pesquisas Epidemiológicas, Universidade Estadual da Paraíba, Av. das Baraúnas, 351, \\ Bodocongó, 58109-753 Campina Grande-PB, Brasil
}

\begin{abstract}
RESUMO: Há muito tempo as propriedades sedativas do maracujazeiro já são conhecidas, especialmente quando utilizadas as infusões ou tinturas das folhas. Recentemente foi descrita uma nova propriedade relacionada ao fruto: a atividade hipoglicemiante da farinha produzida a partir de sua casca, porção rica em pectina, uma fração de fibra solúvel que, quando hidratada, regula respostas metabólicas na redução de absorção de nutrientes, como glicídios e lipídios, devido à formação de uma camada gelatinosa na mucosa intestinal, mecanismo que pode explicar sua ação hipoglicemiante. O objetivo desse estudo foi avaliar a toxicologia clínica do uso da farinha de casca de maracujá em voluntários saudáveis. Para isso foi realizado um ensaio toxicológico clínico, em 36 voluntários de ambos os sexos, que utilizaram $10 \mathrm{~g}$ do produto três vezes ao dia, durante oito semanas. Os indivíduos foram incluídos no estudo após uma avaliação clínica, com a realização de exames físicos e laboratoriais. $\mathrm{O}$ uso do produto foi bem tolerado pelos voluntários, não sendo relatadas reações adversas que pudessem comprometer sua utilização como alimento com propriedade de saúde. Os exames efetuados não evidenciaram sinais de toxicidade nos diversos órgãos e sistemas avaliados, indicando que a farinha de casca de maracujá poderá ser utilizada em futuros estudos farmacológicos clínicos.
\end{abstract}

Unitermos: Passiflora edulis, maracujá, cascas, farinha, alimento funcional, toxicologia.

\begin{abstract}
Tests of clinical toxicology of the yellow passion fruit peel (Passiflora edulis, $\mathrm{f}$. flavicarpa), as food with health property". For a long time the sedative properties of passion tree have already been known, particularly when using the infusion or tincture of the leaves. Recently, however, a property related to a new product was described: a hypoglycaemic activity of the flour produced from its bark, which is rich in pectin, a fraction of soluble fiber that lowers the absorption of carbohydrates in our body, which explains its hypoglycaemic mechanism of action. The objective of this study was to evaluate the clinical toxicology of the use of the meal in healthy volunteers. For this purpose a phase 1 clinical toxicological test was performed in 36 volunteers of both sexes, which used $10 \mathrm{~g}$ of the product three times a day for eight weeks. Individuals were included in the study after a clinical evaluation, with the completion of physical examinations and laboratory. The use of the product was well tolerated by volunteers and adverse reactions that could jeopardize their use as food with ownership of health were not reported. The examinations performed revealed no signs of toxicity in various organs and systems evaluated, indicating that the product may be used in future clinical pharmacological studies.
\end{abstract}

Keywords: Passiflora edulis, passion fruit, peels, flour, functional food, toxicology.

\section{INTRODUÇÃO}

Com o aumento acentuado de enfermidades crônicas durante o século XX, tais como o Diabetes mellitus, as doenças cardiovasculares e outras, houve interesse em estudar as populações onde a prevalência dessas enfermidades é baixa. Diversos estudos epidemiológicos indicaram que hábitos de vida, 
especialmente os alimentares, eram fatores de proteção. A partir daí, diversos estudos apontaram para a ação benéfica de componentes alimentares (Ferrari \& Torres, 2003; Lottenberg et al., 2002).

Os alimentos utilizados para manter ou promover a saúde têm recebido denominações diversas, tais como alimentos funcionais, nutracêuticos, alimentos planejados, protetores, bioativos, entre outros. Apesar das diferentes denominações, qualquer alimento que adicionado à dieta natural cause efeito positivo à saúde, pode ser incluído nessa categoria. Nos últimos anos têm crescido o interesse por alimentos ou componentes alimentares ativos fisiologicamente, que promovam a saúde (Chaudahari, 1999; Kwak \& Jukes, 2001).

Para que um alimento seja considerado como funcional, é preciso que os benefícios sobre uma ou mais funções orgânicas sejam satisfatoriamente demonstradas, tanto para a promoção de saúde e bemestar quanto para a redução de riscos de doenças. Os alimentos funcionais devem permanecer como alimentos, e seus efeitos devem ser demonstrados em quantidades que possam ser normalmente ingeridas em uma dieta normal (Milner, 2000; Duffy et al., 2001).

Um fator relevante na utilização de produtos de origem natural é sua crescente aceitação pela população em geral, em parte devido à insatisfação quanto ao custo e segurança da medicina convencional, o que contribui, por exemplo, para o crescente consumo de fitoterápicos nos últimos tempos (Nicoletti, 2003; Viggiano, 2005). Medeiros et al. (2007) avaliaram recentemente a eficácia de um medicamento fitoterápico utilizado pela população da Paraíba há mais de cem anos, e demonstraram que o produto realmente apresenta propriedades antiinflamatórias.

Diversos outros estudos estão sendo realizados com o intuito de descobrir novas propriedades medicinais que os produtos naturais possam possuir, e não só para tratar enfermidades crônicas relacionadas ao estilo de vida moderno. Alguns aspectos de pesquisas com produtos naturais direcionados à produção de drogas contra a hanseníase foram discutidos por Barbosa-Filho et al. (2007), em uma revisão recente sobre produtos naturais com atividade antileprótica.

As propriedades sedativas do maracujá são conhecidas há muito tempo, especialmente quando se utiliza a infusão ou tintura das folhas (Guertzenstein, 1998; Oliveira et al., 2002). Recentemente, porém, foi descrita uma nova propriedade relacionada ao fruto: a atividade hipoglicemiante da farinha produzida a partir de sua casca (Guertzenstein, 1998; Petry et al., 2001).

A casca de maracujá é rica em pectina, uma fração de fibra solúvel capaz de ligar-se à água e formar compostos de alta viscosidade, conferindo-lhe efeitos fisiológicos peculiares (Sanderson, 1981). A hidratação da fibra ocorre pela adsorção de água à sua superfície ou pela incorporação ao interstício macromolecular (Schweizer \& Wursch, 1991). Na mucosa intestinal há formação de uma camada gelatinosa, que altera a difusão e absorção de nutrientes. Em função dessa maior viscosidade do conteúdo entérico, efeitos críticos regulam a resposta metabólica à carga de nutrientes (Brown et al., 1979; Schneeman, 1986), como por exemplo, o decréscimo na absorção de carboidratos pelo organismo, mecanismo que pode explicar sua ação hipoglicemiante (Trowell, 1978).

Cascas de maracujá (Passiflora edulis, f. flavicarpa, DEG ) cv amarelo, após desidratadas foram transformadas em farinhas e empregadas como fonte de fibra na formulação de rações para grupos de ratos adultos, sadios e diabéticos. A análise do comportamento da glicemia ao longo do experimento que durou 28 dias demonstrou redução significativa, frente à glicemia dos animais no início do experimento. Os resultados desse estudo sugerem, com eficácia, a utilização da farinha da casca de maracujá cv. amarelo no controle da diabetes, assim como de determinadas patologias, diante da confirmação de tratar-se de um subproduto rico em pectina (fibra solúvel) (Guertzenstein \& Sabaa-Srur, 2002).

Outra pesquisa, realizada com animais experimentais durante 28 dias com dieta contendo fibras insolúveis (Frias \& Sgarbieri, 1998) mostrou significativa redução na concentração de glicose sanguínea. Chandalia et al. (2000) mostraram, em estudos randomizados, que o alto consumo de fibras em geral melhora o controle glicêmico e reduz a hiperinsulinemia em portadores de Diabetes mellitus tipo 2.

Os resultados dessas pesquisas foram amplamente divulgados nos meios de comunicação em massa, a partir de maio de 2003, levando milhares de pessoas a utilizarem a farinha para reduzir os níveis glicêmicos. Após essas divulgações, inúmeras pessoas começaram a produzir de maneira artesanal e consumir e propagar os efeitos benéficos dessa farinha sobre a diabete. As empresas não tardaram a fabricar a farinha da casca do maracujá em escala industrial e a iniciar sua venda.

Uma extensa revisão realizada por BarbosaFilho et al. (2005) sobre plantas e seus constituintes com atividade hipoglicemiante das Américas do Sul, Central e do Norte não relaciona o Passiflora edulis como um produto vegetal com esta propriedade. Por outro lado, um estudo recente sobre as plantas conhecidas como medicinais e venenosas do Nordeste do Brasil, citou que o Passiflora edulis está sendo utilizado na forma de alimento para combater o diabetes, além do uso tradicional de seu suco como sedativo (Agra et al., 2007; 2008).

Por outro lado, produtos de origem vegetal podem apresentar diversos efeitos adversos; por isso vários estudos de toxicologia clínica têm sido realizados, com o objetivo de demonstrar a segurança do uso destes produtos (Soares et al., 2006; Tavares et al., 2006). 
Ainda não existem evidências científicas de que a farinha da casca do maracujá possa realmente ter uma ação hipoglicêmica em humanos e, especialmente, se seu uso é isento de efeitos tóxicos para o organismo. Diniz et al. (1998) afirmam que suas folhas, assim como o fruto imaturo, possuem glicosídeos cianogênicos em sua composição, capazes de provocar distúrbios respiratórios. Dessa forma, esse trabalho se propõe a avaliar a segurança do uso do maracujá-amarelo (Passiflora edulis, f. flavicarpa), utilizado na forma de farinha como complemento alimentar, através de ensaios toxicológicos clínicos, fase 1, em voluntários saudáveis.

\section{MATERIAL E MÉTODOS}

\section{Desenho do experimento}

O estudo consistiu de um ensaio toxicológico clínico aberto, não-controlado, com 36 voluntários saudáveis, com idades entre 20 e 60 anos, sendo 20 mulheres e 16 homens. Cada voluntário serviu como seu próprio controle, pois os dados obtidos na avaliação basal foram comparados com aqueles obtidos após o uso do produto alimentício.

Como produto alimentício foi utilizada a farinha do albedo (casca) do Passiflora edulis, f. flavicarpa produzida pela A. S. S. Neto's Alimentos Ltda (CNPJ 82.112.708/0001-20) localizada no Rio de Janeiro sob a orientação do Professor Dr. Armando Ubirajara Oliveira Sabaa Srur.

\section{Seleção dos indivíduos}

Os voluntários foram submetidos a uma avaliação clínica e laboratorial para serem considerados aptos a participar da pesquisa. Foram excluídos aqueles sujeitos considerados inaptos durante a anamnese e/ou o exame físico, ou demonstraram alterações laboratoriais nos exames de análises clínicas que revelassem disfunção hepática ou renal, intolerância aos carboidratos ou Diabetes mellitus. Também foram excluídos voluntários com hipertensão arterial sistêmica, portadores de alterações cardíacas, gestantes, alcoolistas ou que estavam em uso de alguma medicação hipoglicemiante, medicações de uso contínuo ou ainda que estivessem realizando dietas especiais.

\section{Protocolo experimental e considerações éticas}

Os voluntários foram orientados a comparecer ao Centro de Endocrinologia e Metabologia Ltda, na cidade de Campina Grande-PB para a avaliação inicial, que verificou as funções hepática, renal e cárdiorespiratória, através da história clínica, exame físico e os exames laboratoriais que antecederam o estudo. Os voluntários foram orientados a ingerir $10 \mathrm{~g}$ do produto alimentício de origem vegetal em estudo, três vezes ao dia, diariamente durante 8 semanas. Para ingestão desse produto, os voluntários deveriam adicioná-lo a um suco, refresco, sopa ou outro alimento. Foram avaliados os efeitos da administração da farinha do albedo de Passiflora edulis no terceiro e no sétimo dia da administração diária, para verificação de toxicidade aguda. Os indivíduos foram avaliados ainda na terceira e na sexta semanas da administração diária e 24 horas após a última dose (oitava semana), para avaliação da toxicidade crônica. Durante todo o curso dos experimentos, os voluntários foram instruídos a registrar e comunicar ao pesquisador quaisquer sinais ou sintomas adversos que porventura ocorressem.

$\mathrm{O}$ projeto que deu origem a esta pesquisa foi encaminhado e aprovado pelo Comitê de Ética em Pesquisa (CEP) da Universidade Estadual da Paraíba. Foram seguidas neste estudo as recomendações da resolução 196/96 do Conselho Nacional de Saúde (CNS), que normatiza pesquisas em seres humanos, e a Resolução no 18/99 da Agência Nacional de Vigilância Sanitária (ANVISA), que estabelece as diretrizes básicas para análise e comprovação de propriedades funcionais e ou de saúde alegadas aos alimentos. A metodologia do experimento foi explicada aos voluntários, que assinaram o termo de consentimento de participação livre e esclarecido.

\section{Análise estatística}

Os resultados dos experimentos foram submetidos à análise estatística através do software SPSS (Statistical Package for the Social Sciences) versão 11. Foram utilizados média e desvio-padrão, análise de variância (ANOVA) e teste $\mathrm{t}$ de Student, sendo posteriormente apresentados em tabelas. O limiar significativo (nível de significância) foi fixado em $95 \%$ em todos os casos.

\section{RESULTADOS E DISCUSSÃO}

Foram convidados quarenta e sete (47) indivíduos, sendo vinte e dois (22) homens e vinte e cinco (25) mulheres. Depois de aplicados os critérios de exclusão, trinta e seis (36) voluntários chegaram ao término do experimento, sendo dezesseis (16) homens e vinte (20) mulheres. A idade média dos voluntários foi de 36,88 \pm 10,55 anos. Em relação ao peso, verificou-se uma redução média de $2 \%$ entre a avaliação basal e a avaliação final, após oito semanas do uso da farinha do albedo de Passiflora edulis. O valor médio inicial foi de $72,75 \pm 10,85 \mathrm{~kg}$ e o valor médio final foi de 71,30 $\pm 9,91 \mathrm{~kg}$.

O uso do produto foi bem tolerado, havendo relatos isolados de efeitos adversos. Entretanto, nenhum dos eventos foi relacionado com a administração da farinha. Os exames efetuados não evidenciaram sinais de toxicidade nos diversos órgãos e sistemas avaliados, 
Tabela 1. Resultados dos exames laboratoriais obtidos dos indivíduos que utilizaram a farinha da casca de Passiflora edulis, antes e após o término da administração, por gênero.

\begin{tabular}{|c|c|c|c|c|}
\hline \multirow{2}{*}{ Parâmetros laboratoriais } & \multicolumn{2}{|c|}{$\begin{array}{l}\text { Gênero Feminino } \\
\qquad(\mathrm{n}=20)\end{array}$} & \multicolumn{2}{|c|}{$\begin{array}{l}\text { Gênero masculino } \\
\qquad(\mathrm{n}=16)\end{array}$} \\
\hline & $\begin{array}{c}\text { Basal } \\
\text { Média } \pm \text { DP }\end{array}$ & $\begin{array}{c}8 \text { semanas } \\
\text { Média } \pm \text { DP }\end{array}$ & $\begin{array}{c}\text { Basal } \\
\text { Média } \pm \text { DP }\end{array}$ & $\begin{array}{c}8 \text { semanas } \\
\text { Média } \pm \text { DP }\end{array}$ \\
\hline Acido úrico (mg/dL) & $4,80 \pm 1,01$ & $4,52 \pm 0,81$ & $4,48 \pm 1,03$ & $4,31 \pm 0,99$ \\
\hline Albumina (g/dL) & $4,73 \pm 0,43$ & $4,55 \pm 0,40$ & $4,78 \pm 0,42$ & $4,80 \pm 0,44$ \\
\hline $\operatorname{ALT}(\mathrm{U} / \mathrm{L})$ & $26,60 \pm 6,66$ & $25,10 \pm 4,86$ & $27,06 \pm 5,54$ & $26,87 \pm 5,72$ \\
\hline Amilase (U/L) & $80,85 \pm 22,54$ & $75,60 \pm 19,66$ & $75,06 \pm 17,29$ & $83,37 \pm 20,35$ \\
\hline AST (U/L) & $27,80 \pm 6,30$ & $28,00 \pm 5,66$ & $28,12 \pm 5,31$ & $27,56 \pm 6,04$ \\
\hline Bilirrubina (mg/dL) & $0,50 \pm 0,23$ & $0,64 \pm 0,20$ & $0,69 \pm 0,21$ & $0,67 \pm 0,19$ \\
\hline Cálcio (mg/dL) & $10,24 \pm 0,38$ & $10,00 \pm 0,53$ & $10,18 \pm 0,42$ & $10,15 \pm 0,34$ \\
\hline Creatinina (mg/dL) & $0,82 \pm 0,01$ & $0,86 \pm 0,16$ & $0,81 \pm 0,02$ & $0,81 \pm 0,17$ \\
\hline CPK (U/L) & $106,00 \pm 23,53$ & $106,10 \pm 28,78$ & $107,43 \pm 24,16$ & $107,68 \pm 17,14$ \\
\hline Fosfatase alcalina (U/L) & $79,35 \pm 25,60$ & $75,65 \pm 24,43$ & $80,93 \pm 30,33$ & $84,43 \pm 33,68$ \\
\hline Gama GT (U/L) & $26,70 \pm 7,68$ & $28,65 \pm 7,26$ & $28,25 \pm 8,56$ & $26,43 \pm 8,05$ \\
\hline Glicemia (mg/dL) & $83,95 \pm 6,25$ & $82,00 \pm 6,92$ & $85,43 \pm 6,03$ & $84,37 \pm 6,70$ \\
\hline Hemácias $\left(\mathrm{x} 10^{6} / \mathrm{mm}^{3}\right)$ & $4,48 \pm 0,34$ & $4,49 \pm 0,31$ & $5,00 \pm 0,42$ & $5,03 \pm 0,39$ \\
\hline Hematócrito (\%) & $40,00 \pm 1,91$ & $39,93 \pm 1,63$ & $43,60 \pm 3,05$ & $43,75 \pm 2,10$ \\
\hline Hemoglobina (g/dL) & $13,00 \pm 0,91$ & $13,03 \pm 0,64$ & $14,58 \pm 0,88$ & $14,52 \pm 0,90$ \\
\hline LDH (U/L) & $319,95 \pm 67,59$ & $307,20 \pm 71,46$ & $319,68 \pm 60,72$ & $337,25 \pm 50,94$ \\
\hline Leucócitos $\left(\mathrm{x} 10^{3} / \mathrm{mm}^{3}\right)$ & $6,94 \pm 1,12$ & $6,92 \pm 1,09$ & $6,81 \pm 0,76$ & $6,87 \pm 0,85$ \\
\hline Plaquetas $\left(\mathrm{x} 10^{3} / \mathrm{mm}^{3}\right)$ & $263,75 \pm 80,15$ & $268,85 \pm 81,75$ & $249,37 \pm 86,22$ & $248,06 \pm 81,41$ \\
\hline Potássio (Mmol/L) & $4,45 \pm 0,59$ & $4,47 \pm 0,57$ & $4,57 \pm 0,31$ & $4,58 \pm 0,30$ \\
\hline Proteína total (mg/dL) & $6,42 \pm 1,04$ & $6,69 \pm 0,95$ & $6,63 \pm 0,85$ & $6,46 \pm 0,98$ \\
\hline Sódio (Mmol/L) & $143,60 \pm 3,06$ & $144,55 \pm 2,50$ & $143,56 \pm 3,57$ & $143,25 \pm 2,35$ \\
\hline Uréia $(\mathrm{mg} / \mathrm{dL})$ & $28,85 \pm 7,22$ & $28,65 \pm 7,42$ & $29,62 \pm 7,86$ & $29,87 \pm 7,76$ \\
\hline
\end{tabular}

pois não foram observadas variações estatisticamente significantes $(\mathrm{p}>0,05)$ quando se comparou a avaliação do período basal com a avaliação realizada após o término da administração (Tabela 1), o que sugere que o produto poderá ser utilizado em estudos farmacológicos.

Ensaios toxicológicos clínicos são importantes para avaliar a segurança de fármacos. São realizados com uma pequena amostra de voluntários saudáveis, podendo seu número variar de 20 a 80 , de ambos os sexos (Moraes et al., 2004). Por se tratar de um produto que já vinha sendo utilizado pela população, adotou-se a dosagem preconizada pela maioria dos fabricantes desse produto alimentício, que é de três doses diárias de $10 \mathrm{~g}$ cada.

Dentre os parâmetros bioquímicos utilizados, as provas de função hepática desempenham importante interesse, pois diversas substâncias são extensamente biotransformadas pelo fígado. As bilirrubinas são resultantes do catabolismo da hemoglobina, após a destruição normal ou patológica das hemácias. As transaminases (ALT e AST) e a gama GT são bons marcadores, pois ocorre seu aumento em $90 \%$ dos casos de doença hepática (Lima et al., 2002).

A dosagem de creatinina é um importante sinalizador clínico, já que a queda da filtração glomerular com conseqüente elevação da taxa de creatinina plasmática implica na perda da função renal
(Berne \& Levy, 2006). Além disso, o valor plasmático da creatinina sofre influências da massa muscular, nutrição e ocorrência de edema (Soares et al., 2002).

O hemograma é um importante aliado dos estudos de toxicidade, pois o sistema hematopoiético é extremamente sensível a atividades de agentes tóxicos, principalmente aqueles com potencial mutagênico ou citotóxico, resultando em alterações qualitativas ou quantitativas, transitórias ou permanentes e que podem limitar a utilização de fármacos e medicamentos; alterações hematológicas podem refletir, também, na atividade imunológica (Lima et al., 2002).

Durante as avaliações intermediárias, realizadas no terceiro e no sétimo dias, assim como na terceira e sexta semanas de administração do produto, foram observadas pequenas variações nos parâmetros bioquímicos e hematológicos avaliados. Porém estas variações mantiveram-se dentro dos valores de referência para cada analito. Esse ensaio clínico demonstrou que o uso da farinha do albedo de Passiflora edulis pode ser testado com segurança para avaliação de sua eficácia em pacientes portadores de diabetes, assim como naqueles portadores de dislipidemias. Entretanto, esses resultados não garantem ausência de reações adversas, especialmente aquelas de baixa incidência, que somente seriam observadas após utilização por centenas ou milhares de pacientes. Desse modo, é importante manter 
um programa de farmacovigilância durante a fase de comercialização do produto (Ferreira, 2006; Silveira et al., 2008).

Pode-se concluir que a administração da farinha do albedo de Passiflora edulis, na dose preconizada, mostrou-se segura, sem apresentar alterações que pudessem comprometer seu uso como alimento com propriedade de saúde.

\section{AGRADECIMENTOS}

Este estudo contou com o apoio da Coordenação de Aperfeiçoamento de Pessoal de Nível Superior (CAPES) e da A.S.S. Neto's Alimentos Ltda.

\section{REFERÊNCIAS}

Agra MF, Freitas PF, Barbosa-Filho JM 2007. Synopsis of the plants known as medicinal and poisonous in Northeast of Brazil. Rev Bras Farmacogn 17: 114-140.

Agra MF, Silva KN, Basílio IJLD, França PF, Barbosa-Filho JM 2008. Survey of medicinal plants used in the region Northeast of Brazil. Rev Bras Farmacogn 18: 472508.

Barbosa-Filho JM, Vasconcelos THC, Alencar AA, batista LM, Oliveira RAG, Guedes DN, Falcão HS, Moura MD, Diniz MFFM, Modesto-filho J 2005. Plants and their active constituents from South, Central, and North America with hypoglycemic activity. Rev Bras Farmacogn 15: 392-413.

Barbosa-Filho JM, Nascimento-Júnior FA, Tomaz ACA, Athayde-Filho PF, Silva MS, Cunha EVL, Souza MFV, Batista LM, Diniz MFFM 2007. Natural products with antileprotic activity. Rev Bras Farmacogn 17: 141148.

Berne RM, Levy MN 2006. Fisiologia. 4. ed. Rio de Janeiro: Guanabara Koogan, p. 253.

Brown RC, Kelleher J, Losowsky MS 1979. The effect of pectin on the structure and function of the rat small intestine. Brit J Nutr 42: 357-65.

Chandalia M, Garg A, Lutjohann D, Bergaman KV, Grundy SM, Brinkley LJ 2000. Beneficial effects of high dietary fiber intake in patients with type 2 diabetes mellitus. New Engl J Med 342: 1392-1398.

Chaudahari RF 1999. Foods of the future: the impact of functional foods in the cereal industry, Cereal Food World 44: 93-5.

Diniz MFFM, Oliveira RAG, Medeiros ACD, Malta Júnior A 1998. Memento fitoterápico - As plantas como alternativa terapêutica: aspectos populares $e$ cientificos. João Pessoa: ed. Universitária/UFPB. P.119-122.

Duffy SJ, Keaney-Junior JF, Holbrook M, Gokce N, Swerdloff PL, Frei B, Vita JA 2001. Short and long-term black tea consumption reverses endothelial dysfunction in patients with coronary artery disease. Circulation 104: 151-156.

Ferrari CKB, Torres EA 2003. Biochemical pharmacology of functional foods and prevention of chronic disease of aging. Biomed Pharmacother 57: 251-260.

Ferreira EI 2006. Como nascem e se desenvolvem os novos medicamentos. In: Silva P. Farmacologia. 7. ed. Rio de janeiro: Guanabara Koogan. p.213-221.

Frias AD, Sgarbieri VC 1998. Guar gum effects on blood serum lipids and glucose concentrations of wistar diabetic rats. Cienc Tecn Alim 18: 60-62.

Guertzenstein SMJ 1998. Caracterização da farinha da casca de maracujá (Passiflora edulis, f. flavicarpa, DEG) cv. amarelo como fonte de fibra solúvel para alimentação humana. Rio de Janeiro, 221 p. Dissertação (Mestrado em Nutrição) - Universidade Federal do Rio de Janeiro.

Guertzenstein SMJ, Srur AUOS 2002. Uso da casca de maracujá (Passiflora edulis, f. flavicarpa, DEG) cv amarelo na alimentaçãode ratos (rattus norvergicus) normais e diabéticos. Cadernos do Centro Universitário São Camilo 10: 213-218.

Kwak NS, Jukes DJ 2001. Functional Foodas. Part I: The development of a regulatory concept. Food Cont 12: 99-107.

Lima AO, Soares JB, Greco J 2002. Métodos de Laboratório Aplicados à Clínica. 8. ed. Rio de Janeiro: Guanabara Koogan, p. 126-129.

Lottenberg AMP, Nunes VS, Nakandakare ER, Neves M, Bernik M, Santos JE, Quintão ECR 2002. Eficiência dos ésteres de fitoesteróis alimentares na redução dos lipídes plasmáticos em hipercolesterolêmicos moderados. Arq Bras Cardiol 79: 1-4.

Medeiros KCP, Monteiro JC, Diniz MFFM, Medeiros IA, Silva BA, Piuvezam MR 2007. Effect of the activity of the Brazilian polyherbal formulation: Eucalyptus globulus Labill, Peltodon radicans Pohl and Schinus terebinthifolius Radd in inflammatory models. Rev Bras Farmacogn 17: 23-28.

Milner JA 2000. Functional foods: the US perspective. Am J Clin Nutr 71 (Suppl): 1654S-1659S.

Moraes MO, Bezerra FAF, Lotufo LC, Pessoa C, Moraes MEA 2004. Avaliação clínica da eficácia e segurança de fitoterápicos no Brasil. Arq Bras Fitomed Cient 1: 3039.

Nicoletti MA 2003. Fitoterapia: Curcuma zedoaria (Christm) Roscoe, uma possibilidade terapêutica como antifúngico de uso tópico. Pharm Bras 38: 89-91.

Oliveira LF, Nascimento MRF, Borges SV, Ribeiro PCN, Ruback VR 2002. Aproveitamento alternativo da casca do maracujá-amarelo (Passiflora edulis f. flavicarpa) para produção de doce em calda. Cienc Tecnol Aliment 22: 259-262.

Petry RD, Reginato F, De Paris F, Gosmann G, Salgueiro JB, Quevedo, Kapczinski F, Ostega GG, Schenkel EP 2001. Comparative pharmacological study of hydroethanol extracts of Passiflora alata and Passiflora edulis leaves. Phytother Res 15: 162-164.

Sanderson GR 1981. Polysaccharides in Foods. Food Technol 35: 50-57, 83.

Schneeman BO 1986. Dietary fiber: physical and chemical properties,methods of analysis, and physiological effects. Food Technol 40: 104-110.

Schweizer TF, Wursch P 1991. The physiological and nutritional importance of dietary fibre. Experientia 47: 181-186.

Silveira PF, Bandeira MAM, Arrais PSD 2008. Farmacovigilância e reações adversas às plantas medicinais e fitoterápicos: uma realidade. Rev Bras Farmacogn 18: 618-626.

Soares JLMF, Pasqualotto AC, Rosa DD, Leite VRS 2002. Métodos diagnósticos - consulta rápida. Porto Alegre: Artmed Editora.

Soares AKA, Carmo GC, Quental, DP, Nascimento DF, Bezerra, FAF, Moraes MO, Moraes MEA 2006. Avaliação da segurança clínica de um fitoterápico contendo Mikania glomerata, Grindelia robusta, Copaifera officinalis, Myroxylon toluifera, Nasturtium officinale, própolis e mel em voluntários sadios. Rev Bras Farmacogn 16: 
447-454

Srur AUOS, Guertzenstein SMJ 1999. Uso da farinha da casca do maracujá (Passiflora edulis, f.flavicarpa, DEG) na alimentação de ratos adultos da linhagem Wistar (Rattus norvegicus var. Albinus, Rodentis mammalia) diabéticos e normais. Anais do I Congresso Latino Americano de Nutrição Humana, Gramado-RS, p. 95.

Tavares JP, Martins IL, Vieira AS, Lima FAV, Bezerra FAF, Moraes MO, Moraes MEA 2006. Estudo de toxicologia clínica de um fitoterápico a base de associações de plantas, mel e própolis. Rev Bras Farmacogn 16: 350356.

Trowell HC 1978. Diabetes mellitus and dietary fiber of starch foods. Am J Clin Nutr 31: 53-62.

Viggiano CE 2005. A segunda era de ouro da nutrição: alimentos funcionais. Revista de Nutrição Profissional 1: 12-20. 